Methods: Qualitative interpretive design, with 12 eligible participants using semi-structured interviews.

Key Findings: A thematic analysis found three interconnected themes: 'self-efficacy', 'support' and 'sustainability'. Self-efficacy influenced the women's readiness and motivation to breastfeed and discharge home early. Social, semi-professional and professional breastfeeding supports were key in women's experiences. Individualised breastfeeding support and emotional reassurance were identified as important factors in promoting self-efficacy and confidence. A strong connection between the woman's self-efficacy and the breastfeeding support they received was demonstrated. Sustainability referred to and explored what women valued in relation to sustaining their breastfeeding journey. Namely, ongoing accessible breastfeeding supports, from people such as midwives, community nurses and lactation consultants, and from online resources. These resources were experienced as accessible forms of breastfeeding information, emotional support and a sense of community.

Implications for practice: The study highlights the importance of effective breastfeeding support and suggests the need for more evidence-based breastfeeding online resources, potentially in the form of an interactive applications or websites, and that accessible people based breastfeeding services in the community should be increased. Additionally, a focus on holistic and individualised breastfeeding assessments and care plans prior to discharge that link women with ongoing breastfeeding services is paramount.

https://doi.org/10.1016/j.wombi.2018.08.023

07

\section{Evaluation of an innovative approach to enhancing midwives' understanding of breastfeeding physiology through the use of animation}

Nicki Hartney, Cate Nagle, Dolores Dooley

\section{Deakin University, Victoria, Australia}

Background: Supporting women to breastfeed requires knowledge of the physiology, including the complex interplay of both endocrine and autocrine systems. The use of e-learning provides an alternative platform for customising and diversifying content to appeal to a variety of learning styles. While the evidence addressing the effectiveness of instructional animation is equivocal, an innovative approach utilising the application of animation in enhancing midwives' knowledge regarding lactation is warranted. Drawing on expertise in lactation, midwifery education and video design and production, a seven-minute animated video was produced. In this presentation we will showcase the video and present the results of the evaluation.

Methods: The aim of this study was to evaluate midwives' understanding of breastfeeding physiology and the acceptability and usability of the animated resource. In February/March 2018, midwives across Australia were offered the opportunity to review and evaluate the video by completing a cross-sectional anonymous online survey consisting of 29 items containing 4-point Likert scales and free-text responses. Invitation was via the Australian College of Midwives' national newsletter. Ethical approval from Deakin University was obtained. Descriptive statistics were used to analyse quantitative data and free-text was analysed using content analysis.

Results: Results of the registered midwife evaluation will be presented and these will demonstrate midwives' experience of the animation as a learning resource. The presentation will also include midwives' views on how the resource impacted on their breastfeeding knowledge and understanding. Comparison will be made to the midwifery students' evaluation where $85-95 \%$ of respondents reported that the resource enhanced their learning.

Discussion and conclusion: Evidence to inform the use of innovative approaches to support traditional teaching methods will be provided. These results will provide valuable evidence to inform further innovation in the area of professional development education in breastfeeding and other complex midwifery concepts.

https://doi.org/10.1016/j.wombi.2018.08.024

08

It takes a village to raise a child: Improving breastfeeding success in a rural area

Wendy Buckland

Portland District Health, Victoria, Australia

Introduction: The SEA Change Portland is a collective approach to reducing obesity. As part of SEA Change the Breastfeeding Action Plan 2015 was developed through a collaboration between Portland District Health (PDH), Glenelg Shire Council's Maternal and Child Health, Dhauwurd-Wurrung Elderly and Community Health Service Inc. (DWECHS) and Australian Breastfeeding Association (ABA) Portland Group.

Midwives are no strangers to the evidence that shows breastfeeding provides significant value to infants, mothers and society, including the reduction of obesity. However breastfeeding rates in Portland, Victoria have been disappointingly low. Knowing that women need support to initiate and continue breastfeeding, a unique approach to improve that support was initiated.

Aim: The aim of the project was to increase the percentage of babies exclusively breastfed from birth to six months of age. The strategy was to offer all first time mothers booked to birth at Portland District Health an Australian Breastfeeding Association gift membership and one Breastfeeding education class. Once the initiative commenced, Portland District Health made the decision to expand the initiative to include all women who birthed at the organisation, regardless of parity.

Findings: Although a relatively small sample, there was a marked increase in women fully breastfeeding upon hospital discharge between 2015 (50\%) and 2016 (87\%).

Implications for practice: The initiative is the first of its kind in Australia. In addition to the statistical gains the verbal feedback from the women of Portland was encouraging as they surpassed their initial breastfeeding goals, reported feeling more empowered and able to overcome barriers to breastfeeding. The success of the initiative has resulted in a commitment by the Portland District Health to provide ongoing funding to support the project.

https://doi.org/10.1016/j.wombi.2018.08.025

09

Implementing midwifery continuity of care models in NSW regional areas

Elysse Prussing, Graeme Browne, Eileen Dowse, Amanda Wilson

University of Newcastle, New South Wales, Australia

Background: Evidence shows significant improvements in outcomes for women and their babies when they are supported by Midwifery Continuity of Care Models. Despite this evidence and current Australian health directives, widespread implementation of Midwifery Continuity of Care models has not been achieved. This is especially true in regional areas. 